Military Technical College Kobry El-Kobbah, Cairo, Egypt

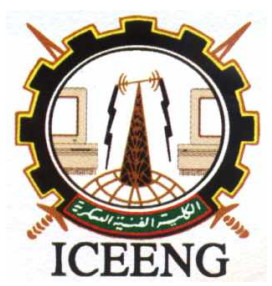

\section{$6^{\text {th }}$ International Conference on Electrical Engineering ICEENG 2008}

\title{
The degree of visibility of the moon phases based on their spatial Observability
}

\author{
By
}

Abdel kawy M.S*

\section{Abstract:}

The spatial observability of the moon phases for different time and places in the world is shown using chaotic time series prediction with fuzzy system. The results are compared with a study using system thinking approach, which show a coincident of both results. Recommendations are proposed for future study to compensate for the moon spatial observability by using spatial state observer. Based on the analogy between moon and sun, using system thinking approach, a proposal is introduced for further study to unify the start of the moon phases.

\section{Keywords:}

Visibility of moon phases, Spatial Observability, lunar seasons, chaotic time series, and fuzzy system. 


\section{Introduction:}

The problem of the moon phase's visibility has a lot of interest throughout the history. In reference [1], this problem is treated as a result of the lunar seasons defined in the same reference. In this paper, this problem will be discussed based on spatial observability of the moon phases. The spatial observability of the moon phases is the observability of the moon system with respect to location of the moon phase's viewer as well as time of view, i.e. the spatial observability depends on space and time. In section 2 , the chaotic time series for the right ascending and descending of the moon will be predicted using fuzzy system. The visibility of the moon phases will be determined based upon two factors. First, is the visibility due to the lunar seasons, which has the cycle of 27.3 days. Second, is the visibility due to the rotation of the earth around its axes? The combination of the two factors, results in determination of the spatial observation of the moon phases as seen in section 3. In this section, the analysis of the results of spatial observation show that the moon phases my not be visible for all viewer in any place in the world for some time in the day, and even for some days in the week. Concluding remarks with analogy based on the system thinking approach, between sun and moon systems are done in section 4. Recommendation and future work are suggested in section 5 .

\section{2. calculation of the visibility of the moon phases :}

\section{1 chaotic time series for the right ascending and descending of the moon}

The point of the moon projection on the earth surface (sub point of the moon) depends on the declination and right ascending of the moon that can be determined from the spatial measurement data on the navigation coordinates [2]. The right ascending of the moon is equal to the longitude of the point of projection of the moon on the earth surface. The descending of the moon is equal to the latitude of the same point. So, in order to predict the observability of the moon for several years, the location of theses points have to be predicted. The fuzzy system with chaotic time series as in reference [1] was used for the calculation of the descending only. In this work, that will be done for both angles.

\subsubsection{Chaotic time series for the right ascending}

The right ascending of the moon can be used together with the proposed fuzzy system to predict the moon position for several years. The design of that system is done through the following steps. (See Mat lab toolbox demo for chaotic time series) 


\subsubsection{Time Series Data}

The data is determined from the measured data in navigation frame transformed to the inertial frame. Based on the results from reference [1], one case will be used with four inputs and as much as available for training data.

\subsubsection{Preprocessing the data}

ANFIS is built, that can predict $x(t+n)$ from the past values of this time series, that is, $x(t-3 n), x(t-2 n), x(t-n)$, and $x(t)$. The time between elements of time series is one hour. The delay between inputs is considered to $b e n=24$, i.e. one day. Therefore the training data format is $[x(t-3 n), x(t-2 n), x(t-n), x(t) ; x(t+n)]$ for the number of inputs $=4$, The number of output is one.

\subsubsection{Building the ANFIS model}

GENFIS1 is used to generate an initial FIS matrix from training data. After ten epochs of training, the final MFs are calculated. Note that these MFs after training do not change drastically. Obviously most of the fitting is done by the linear parameters while the nonlinear parameters are mostly for fine- tuning for further improvement.

\subsubsection{Comparisons}

The difference between the plot of the original time series and the one predicted by ANFIS is so tiny that it is impossible to tell one from another by eye inspection. That is why you probably see only the ANFIS prediction curve. The prediction errors must be viewed on another scale.

\subsubsection{Chaotic time series for the descending}

The same procedures in 2.1.2 will be used to predict the descending of the moon as in [1].

\subsection{Determination of the degree of visibility of moon phases.}

The chaotic time series produced for the moon position in 2.1.1 and 2.1.2 depending on the right ascending and descending of the moon are used to determine the visibility of moon phases using fuzzy system. The fuzzy system has four inputs and one output. The four inputs are the chaotic time series of the right ascending and descending of the moon as well as the longitude and latitude of the location of the viewer. The output of the system is the visibility of the moon phases. It clear from reference [2] that the longitude and latitude of the moon sub point is determined by the right ascending and descending of the moon. The rules for the fuzzy system is constructed based on the fact that the visibility of the moon depends on the difference between longitude and latitude of the moon sub point in one side, and the longitude and latitude of the viewer in the other side. Running the above system on mat lab ANFIS gives the results in figures 2,3,4 for different seasons for three time zones locations on the earth surface which have longitude $=-30,0,30^{\circ}$ respectively . The seasons are determined from the fuzzy system suggested in [1]. These figures are plotted on the world map as shown in figure 1a. Figure $1 \mathrm{~b}$ shows the concentric circles of visibility for the moon for any location of its sub point in the world. 


\section{Analyses of the results of the moon visibility on the world map:}

The results in figure 2, 3 and 4 can be analyzed in two directions. This will be illustrated in the following subsections.

3.1 Horizontally, figure $2 \mathrm{a}, 2 \mathrm{~b}, 2 \mathrm{c}$, show that for the same longitude of the viewer, at different season. The viewer in north can not see the moon phases, whatever the phase is, in the winter season. The viewer in the north can see the moon phases (with different degree of visibility, in summer season. The viewer in the south can not see the moon phases, whatever the phase is, in the summer season (this may be called the southern winter).The same can be seen from figure $3 a, 3 b, 3 c$ and also through figure $4 a, 4 b, 4 c$.

3.2 Vertically, figure $2 \mathrm{a}, 3 \mathrm{a}, 4 \mathrm{a}$; show that for the same season (winter), the viewer at the same latitude will see the moon with the same degree of visibility, but at different time depending on the speed of translation of the sub point of the moon on the earth surface (or on the world map). The same conclusion can be gotten from figure $2 b, 3 b$, $4 \mathrm{~b}$, for spring or autumn, and figure $2 \mathrm{c}, 3 \mathrm{c}, 4 \mathrm{c}$, for summer.

3.3 Regarding the moon phases especially the crescent phase, it is born according to certain criteria according to references [3]-[16]. The moment of appearance of the crescent corresponds to an instance of moon sub point. At this instance, the concentric circles of visibility of the moon phases (in this case the crescent) as shown in figure 1a, will be place at the world map by latitude and longitude corresponding to this instance as seen before ( as time series from section 2).from 3.1 and 3.2 above, one can conclude, that the viewer at the locations in the north, will see the crescent if the season at which the crescent born is summer, in this case it will see it with high degree of visibility. On the other side, the viewer at the locations in the north will not see the crescent if the season at which the crescent born is winter. In this case degree of visibility will be very low till the winter season turns to other one (spring or autumn). The period of any season is near one week (approximately seven days $=27.3 / 4$ ).

\section{Concluding remarks with analogy based on the system thinking approach:}

According to [1] and [17], Natural and universal laws are the basis of systems thinking. One of the fundamental assumptions behind General Systems Theory is that the earth and the systems that comprise it (including human systems like teams and organizations) are governed by natural and universal laws. If we can correctly identify and understand these, we have a better chance of successfully interacting with, and transforming, the systems of which we are a part. Some of the natural and universal laws of life on Earth are four seasons, the life cycle, the sun and the moon (day and night), 365 (plus a few hours. . .) days in a year. Applying this basis on the earth -moon -system, the following analogy between moon and sun as shown in table 1 can be made. 


\begin{tabular}{|l|l|l|}
\hline $\begin{array}{l}\text { Natural and } \\
\text { universal } \\
\text { laws }\end{array}$ & Sun & Moon \\
\hline Four seasons & $\begin{array}{l}\text { Summer, autumn, winter, } \\
\text { and spring }\end{array}$ & $\begin{array}{l}\text { Lunar summer, lunar } \\
\text { autumn, lunar winter, and } \\
\text { lunar spring }\end{array}$ \\
\hline $\begin{array}{l}\text { Effect of the } \\
\text { seasons }\end{array}$ & temperature & $\begin{array}{l}\text { Degree of visibility of the } \\
\text { moon phases }\end{array}$ \\
\hline The life cycle & $\begin{array}{l}\text { Orbit between 23.5 and - } \\
\text { 23.5 latitude }\end{array}$ & $\begin{array}{l}\text { Orbit between } 28.5 \text { and - } \\
\text { 28.5 latitude }\end{array}$ \\
\hline Year (cycle) & 365.25 day & 27.3 days \\
\hline $\begin{array}{l}\text { Definition of } \\
\text { the day and } \\
\text { night: } \\
\text { Time of rise } \\
\text { Time of set }\end{array}$ & $\begin{array}{l}\text { Sun day from sun rise to set } \\
\text { rise } \\
\text { Sun rise (above horizon) } \\
\text { Sun set (down horizon) }\end{array}$ & $\begin{array}{l}\text { Moon day from moon rise to } \\
\text { set } \\
\text { Moon night from moon set to } \\
\text { rise } \\
\text { Moon rise (above horizon) } \\
\text { Moon set (down horizon) }\end{array}$ \\
\hline $\begin{array}{l}\text { Time zones } \\
\text { for events }\end{array}$ & $\begin{array}{l}\text { Time is the same for viewer } \\
\text { at the same longitude } \\
\text { watching an event }\end{array}$ & $\begin{array}{l}\text { Time is the same for viewer } \\
\text { at the same longitude } \\
\text { watching an event }\end{array}$ \\
\hline $\begin{array}{l}\text { Date and } \\
\text { months }\end{array}$ & $\begin{array}{l}\text { Date starts at midnight to } \\
\text { midnight, with fixed months } \\
\text { length }\end{array}$ & $\begin{array}{l}\text { Start of moon months } \\
\text { associated with the detection } \\
\text { of crescent }\end{array}$ \\
\hline
\end{tabular}

Table 1 analogy between moon and sun according to basis of system thinking

From table 1, new concept of the moon seasons and its effect is introduced [1].It is clear that the sun has four seasons which affect the temperature all over the world. This effect is due to the projection of the sun on the world map (earth surface). Summer is one of the four seasons of the year. In the West, the seasons are generally considered to start at the equinoxes and solstices, based on astronomical reckoning. Based on astronomy, summer begins on the day of the summer solstice and ends on the day of the autumn equinox. When it is summer in the in the Northern Hemisphere, it is winter Southern Hemisphere, and vice versa. By other word, when the sun is over the (-23.5) latitude, i.e. in the south, it is summer in the south, and winter on the north. The same can be applied to the moon. When the moon is over (-28.5) latitude it is visible in the south and not visible in the north, (what ever the phase is). In reference [1], a degree of visibility is introduced, (similar to the temperature for the sun) to describe this effect. When the moon is over (-28.5) latitude the degree of visibility of the moon phases is unity for the locations on the earth near (-28.5) latitude, i.e. in the south. 
And it is zero for the locations on the north. The location between the south and north will have the degree of visibility of the moon phases in rang (0-1). So, the degree of visibility of the moon phases (DVMP) depends on the latitude of the moon, as well as the location of the view point (see table 2). DVMP was computed for any location at any time using the fuzzy systems in [1].In addition to that, the definition of the day and night for sun and moon is in table 1. Accordingly, an analogy between length of the day for both sun and moon is shown in table 2. The definition of the day and night for both sun and moon depends on the rotation of the earth from east to west. The length of the day and night depends on the season of sun and moon w.r.t the location of the viewer. The start of the moon month depends on the detection of the crescent as an event. The events is timed for the same longitude for both sun and moon.

\begin{tabular}{|l|l|l|l|l|}
\hline $\begin{array}{l}\text { Effect of } \\
\text { seasons }\end{array}$ & Spring & Summer & Autumn & Winter \\
\hline temperature & Wet & Hot & Dry & Cool \\
\hline $\begin{array}{l}\text { Degree of } \\
\text { visibility of } \\
\text { moon phases }\end{array}$ & $\begin{array}{l}\text { Medium } \\
(0.5) \\
\text { (increasing) }\end{array}$ & High (1) & $\begin{array}{l}\text { Medium } \\
(0.5) \\
(\text { decreasing) }\end{array}$ & Low (0) \\
\hline $\begin{array}{l}\text { Length of the } \\
\text { sun day }\end{array}$ & Medium & long & Medium & short \\
\hline $\begin{array}{l}\text { Length of the } \\
\text { moon day }\end{array}$ & Medium & long & Medium & short \\
\hline $\begin{array}{l}\text { Length of the } \\
\text { sun night }\end{array}$ & Medium & short & Medium & Long \\
\hline $\begin{array}{l}\text { Length of the } \\
\text { moon night }\end{array}$ & Medium & short & Medium & Long \\
\hline
\end{tabular}

Table 2 comparison between temperature and DVMP for sun and moon as well as day length

From table 2, it obvious that the visibility of the both sun and moon depends on the seasons as well as the length of the day and night. The visibility of the moon depends also on the moon phases, taking into consideration that the viewer is with visible light sensor only. The sun is always shining (source of light), so the visibility of the sun is the same as its observability. The moon observability depends on the following:

1) The lunar seasons. 2) The length of the moon day. 3) The moon phase (dark or light phase).

From the above, according to the system thinking approach, the results coincide with the conclusion in chapter 3 . 


\section{Conclusion, recommendation and future work.}

The following are the conclusion, recommendations and future work according to the conclusions from chapter 3 and 4:

1. The moon phases is not observable all the time in any place in the world, therefore it is recommended to use state observer for the moon position during the observable period and place as in reference [18], and use its updated model for predicting the position of the moon during unobservable period and place. That way, the moon position can be measured or predicted for any time at any place. So one can compensate for the spatial Observability of the moon phases, taking into consideration that the data required to calculate the global position of the moon does not depend on any viewer position .By this way the chaotic methods can be used to get higher accuracy, using the accuracy of the data resulting from the observer in short time for unobservable period.

2. Using the available internet to form a network between different sites at different places in the world such that, they include different seasons and different day times, that way a complete GIS spatial data bases can be used as suggested in [2], to have available data for training the chaotic system in [1].

3. From table 1, the time of event of watching the crescent at any place in the world for the first time, is considered to be the same for all viewer of the same longitude. This can be considered as proposal for further study to be used to determine the start of the moon. This may be a start point of study to unify the start of the moon month all over the world.

\section{REFERENCES:}

[1] M. A. Soliman, 6th International Engineering Conference (IEC), Mar2008.

[2] Prof.dr Ibrahim zedan, Dr.M. A. Soliman, and eng.Basheer Nasef. Proc of the 12-th ASAT Conference, 29-31 May 2007 - COMM-07.

[3] Schaefer, B. E., 1988: "Visibility of the Lunar Crescent", Quarterly Journal of the Royal Astronomical Society, Vol. 29, pp. 511-523.

[4] Schaefer, B. E., Ahmad, I. A., Doggett, L. E., 1993: "Records for Young Moon Sightings", Quarterly Journal of the Royal Astronomical Society, Vol. 34, pp. 53-56. [5] Ilyas, M., 1994: "Lunar Crescent Visibility Criterion and Islamic Calendar", Quarterly Journal of the Royal Astronomical Society, Vol. 35, pp. 425-461.

[6] Doggett, L. E., Schaefer, B. E., 1994: "Lunar Crescent Visibility", Icarus, Vol. 107, pp. 388-403.

[7] M. B. Pepin, 1996: "In Quest of the Youngest Moon", Sky \& Telescope, December 1996, pp. 104-106.

[8] Fatoohi, L.J., Stephenson, F.R., and Al-Dargazelli, S.S. 1999, Journal History Astronomy, 30, 51. 
[9] Fatoohi, L.J., Stephenson, F.R., and Al-Dargazelli, S.S. 1998, Observatory, 118, 65. [10] Ilyas, M. 1987, IAU Colloq. 91, 147.

[11] Loewinger, Y. 1995, QJRAS, 36, 449.

[12] McPartlan, M.A. 1996, QJRAS, 37, 837.

[13] Schaefer, B.E. 1990, LunarCal, Western Research Co. Inc., 2127 E. Speedway, Suite 209, Tucson, AZ 85719.

[14] Schaefer, B.E. 1993, Vistas in Astro. 36, 311.

[15] Schaefer, B.E. 1996, QJRAS, 37, 759.

[16] Yallop, B.D. 1997, RGO NAO Tech. Note 69.

[17] Stephen G. Haines, Gail Aller-Stead, and James McKinlay: superior results through systems thinking (2005 by John Wiley \& Sons, Inc.)

[18] ] Prof.dr Ibrahim zedan, Dr.M. A. Soliman, and eng.Basheer Nasef, 6th International Engineering Conference (IEC), Mar2008. 


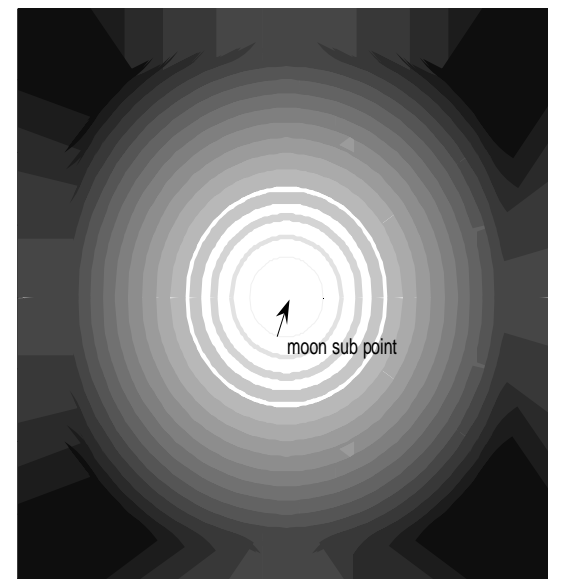

a) Visibility concentric circles where moon sub point at the center assumed $100 \%$ visibility

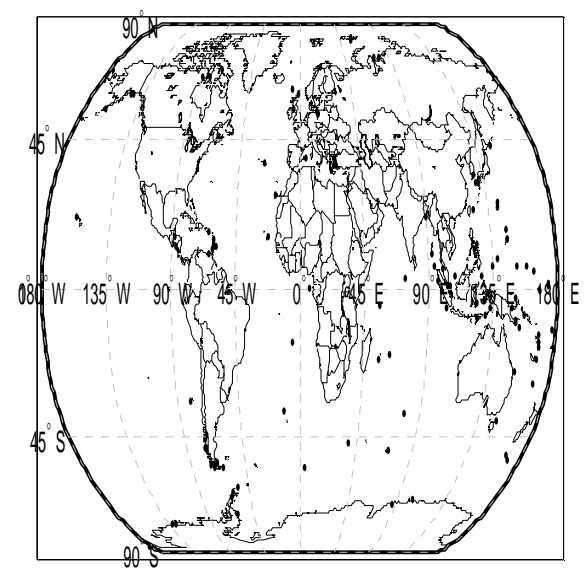

b) world map with

Figure 1 world map and visibility concentric circles where the center placed at moon sub point as shown in next figures

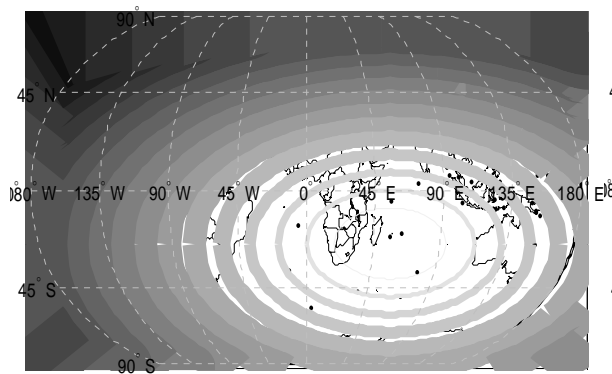

a) Winter

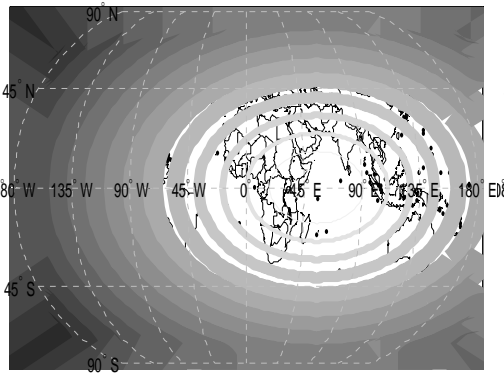

b) spring or autumn

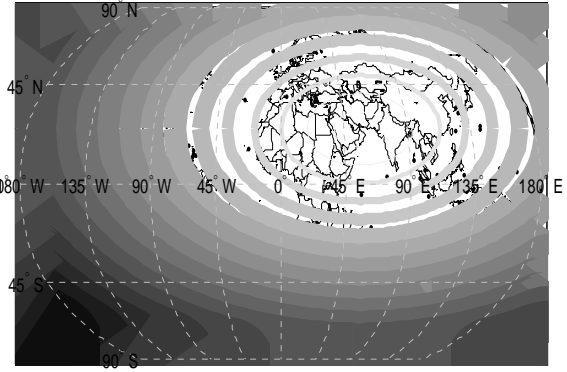

c) summer

Figure 2 moon sub point at -30degree from GMT Greenwich Mean Time at different seasons 


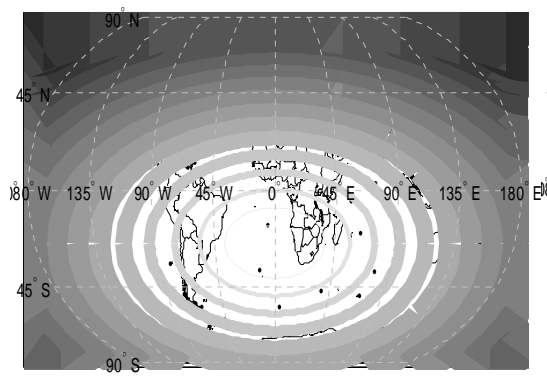

a) Winter

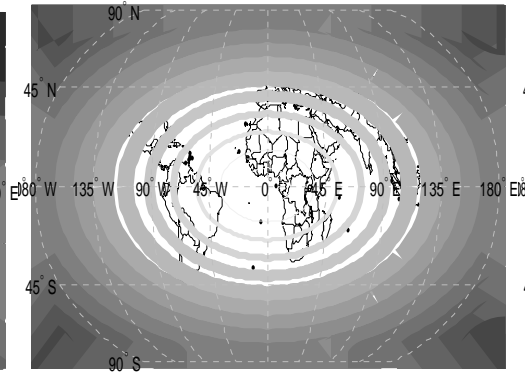

b) spring or autumn

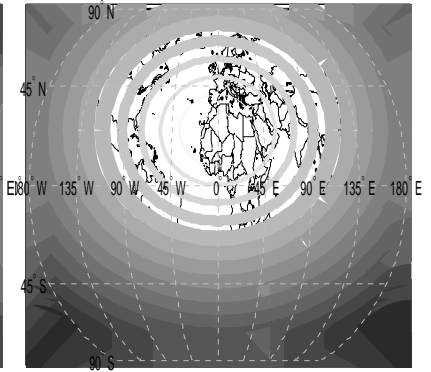

c) summer

Figure 3 moon sub point at GMT Greenwich Mean Time at different seasons

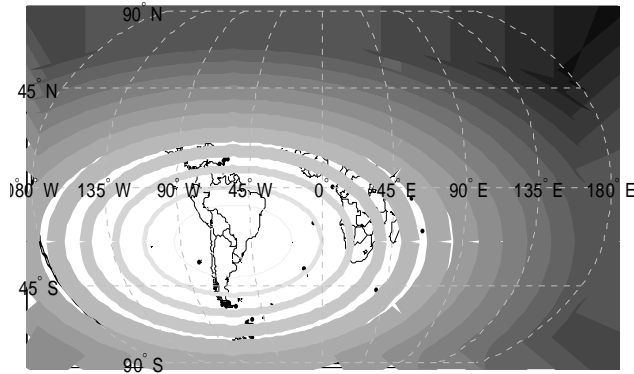

a) Winter

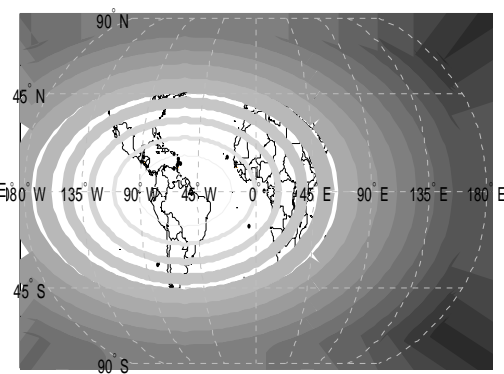

b) spring or autumn

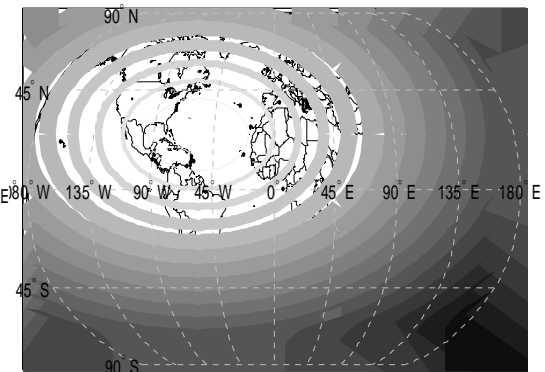

c) summer

Figure 4 moon sub point at +30 degree from GMT Greenwich Mean Time at different seasons 